\section{Cirugía en el paciente cirrótico: características clínicas y complicaciones en una cohorte de pacientes chilenos}

\author{
ABRAHAM IJ GAJARDO ${ }^{1,2}$, RAFAEL PONIACHIK ${ }^{3}$, \\ ALEJANDRO FREUNDLICH ${ }^{3}$, DANIELA B. VERA ${ }^{1}$, \\ CATERINA CHESTA ${ }^{2}$, JAIME RAPPOPORT ${ }^{4}$, JUAN CARLOS DÍAZ ${ }^{4}$, \\ ALEXANDRE SAURE ${ }^{4}$, JAIME CASTILLO ${ }^{4}$, HANS LEMBACH ${ }^{4}$, \\ KATHERINE GONZÁLEZ', CÉSAR NAVEA ${ }^{1}$, JAIME PONIACHIK ${ }^{1}$
}

\section{Surgical complications in cirrhotic patients. Analysis of 102 cases}

Background: Cirrhotic patients have an increased surgical risk due to potential intra and postoperative complications. Aim: To describe the clinical characteristics and surgical complications of cirrhotic patients undergoing surgery in a Chilean university hospital. Patients and Methods: Review of medical records of 102 cirrhotic patients aged $60 \pm 11$ years (52\% males) who underwent elective or urgency surgery at an university hospital between 2010 and 2016. General, pre-surgical, and post-surgical complications were recorded. Results: The main etiologies of cirrhosis were non-alcoholic steatohepatitis (31\%), and alcoholic cirrhosis (28\%). Child-Pugh scores were A, B and C in 50, 28 and $22 \%$ of cases respectively. Median MELD (Model for End-stage Liver Disease) score was 11 (interquartile range: 10-15). The surgical procedure was elective in $71 \%$ of cases, with predominance of abdominal surgery ( $86 \%)$. The American Society of Anesthesiologists (ASA) score was three or more in $52 \%$ of patients. The frequency of any adverse outcome was $62 \%$. The frequency increased along with the severity of cirrhosis and when surgery was urgent. The most common complications were acute renal failure (24\%), increased ascites (23\%) and encephalopathy (22\%). Admission to intensive care unit occurred on $26 \%$ of patients, with six hospital deaths. Conclusions: In these patients, surgical complications were common, although with low mortality.

(Rev Med Chile 2019; 147: 1099-1106)

Key words: Intraoperative Complications; Liver Cirrhosis; Risk Factors.

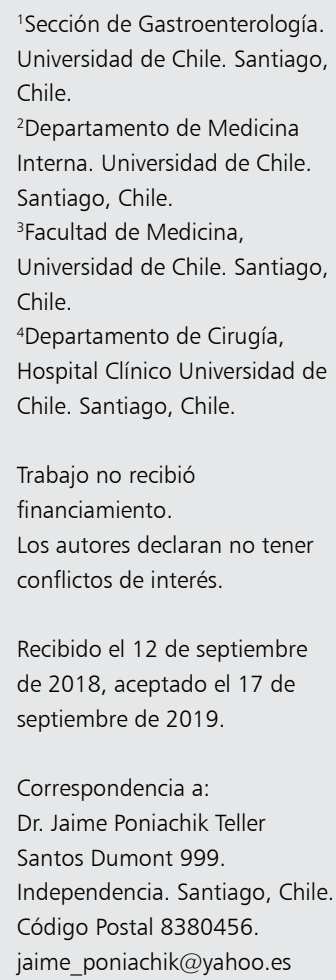

$\mathrm{E}$ 1 paciente cirrótico presenta un riesgo quirúrgico aumentado por las potenciales complicaciones intra y postoperatorias ${ }^{1-4}$. Las razones para una mayor proporción de desenlaces adversos son múltiples y se han relacionado con condiciones inherentes a la cirrosis, reflejo de un equilibrio inestable ante el daño quirúrgico como: hipertensión portal, circulación hiperdinámica, disfunción diastólica, hipoperfusión hepática, riesgo aumentado de infecciones y sangrado, disminución del metabolismo hepático de los fármacos, entre otras ${ }^{2,5}$.

El riesgo quirúrgico de los cirróticos depende del grado de disfunción hepática, hipertensión portal, tipo de procedimiento a realizar y presencia de comorbilidades ${ }^{2,4,6-9}$. Así, constituye un desafío para el equipo médico definir cuáles son los mejores candidatos para ser intervenidos y quiénes son 
adecuados para intervenciones menos invasivas y cuidados postoperatorios especiales por el elevado riesgo quirúrgico ${ }^{2,4}$.

Las escalas MELD y Child-Pugh, junto con cuantificar la gravedad de la enfermedad hepática, pueden ser útiles para definir quiénes podrían acceder a cirugía electiva ${ }^{2-4,7,9,10}$. De esta forma, pacientes con Child-Pugh A o MELD menor a 10 puntos, en general, no tienen contraindicación quirúrgica; aquellos con Child-Pugh B o MELD entre 10 y 15 puntos tienen contraindicación relativa, y finalmente, aquellos con Child-Pugh C o MELD mayor a 15 tienen contraindicación para cirugía electiva, por lo que deben acceder solo en caso de urgencia vital ${ }^{3}$.

La cantidad de pacientes con enfermedad hepática se ha incrementado sostenidamente en la última década, tanto en Latinoamérica como en el mundo ${ }^{11}$, cada día son más los cirróticos sometidos a procedimientos quirúrgicos. Particularmente en Latinoamérica, el restringido acceso a trasplante hepático ${ }^{12}$ condicionaría una mayor prevalencia de cirróticos con enfermedad avanzada y comorbilidades ${ }^{13}$, quienes tendrían alto riesgo quirúrgico y de mortalidad ${ }^{14}$. Sin embargo, son escasos los estudios realizados en países latinoamericanos en los que se evalúa la frecuencia de complicaciones postoperatorias de los cirróticos.

El objetivo primario del estudio es describir las complicaciones quirúrgicas y características clínicas de cirróticos sometidos a cirugía en un hospital universitario de Santiago, Chile, en el período entre 2010 y 2016 . El objetivo secundario es comparar exploratoriamente la frecuencia de desenlaces adversos de acuerdo a la gravedad de la cirrosis y tipo de cirugía.

\section{Métodos}

\section{Diseño del estudio}

Estudio de cohorte retrospectivo, en el cual se incluyeron los pacientes con antecedente de cirrosis que fueron sometidos a cirugía abdominal o de urgencia en el Hospital Clínico Universidad de Chile (Santiago, Chile), en el período 2010-2016. Se excluyeron sujetos cuya primera cirugía fue trasplante de órgano sólido, aquellos sometidos a cirugías de urgencias urológicas, gineco-obstétricas, otorrinológicas, traumatológicas y oftalmológicas, como también los pacientes diagnosticados como cirróticos luego de la cirugía índice.

De acuerdo a la práctica habitual de nuestro centro, las cirugías electivas fueron realizadas por equipos quirúrgicos de subespecialidad, mientras que las de urgencia fueron realizadas por el equipo quirúrgico de turno (compuesto por un cirujano subespecialista, un cirujano general y residentes de cirugía).

\section{Características registradas}

Para construir la cohorte se accedió al registro electrónico de las cirugías mencionadas, identificando a los pacientes que cumplían los criterios de inclusión/exclusión. Médicos y estudiantes de medicina entrenados revisaron los sistemas electrónicos institucionales con el fin de registrar para cada sujeto sus características generales, clínicas pre, intra y postoperatorias.

Las características generales consideradas fueron demográficas, presencia de comorbilidades y etiología de la cirrosis. Como características prequirúrgicas se incluyeron presencia de ascitis, encefalopatía hepática, falla renal (creatinina $>1,5 \mathrm{mg} / \mathrm{dL}$ al ingreso o diálisis), parámetros de laboratorio preoperatorio, puntaje Child-Pugh y MELD y clasificación de riesgo anestésico según la Sociedad Americana de Anestesiólogos (ASA, American Society of Anesthesiologists).

Las características intraoperatorias registradas fueron tipo de cirugía, diagnóstico prequirúrgico, necesidad de cirugía de urgencia o electiva, tipo de anestesia, sangrado intraoperatorio y requerimiento de transfusión de hemocomponentes. Como características postoperatorias se contemplaron el requerimiento de manejo en Unidad de Paciente Crítico (UPC), falla ventilatoria grave (definida por la necesidad de soporte ventilatorio), indicación de diálisis y otras complicaciones postoperatorias como: sangrado mayor (variceal y no variceal), infección, hepatitis isquémica, deterioro de ascitis, deterioro de encefalopatía, falla renal (aumento de creatinina sérica en $0,3 \mathrm{mg} / \mathrm{dL}$ o más), necesidad de reintervención quirúrgica, trasplante hepático, muerte y su causa.

\section{Análisis estadístico}

La determinación de la distribución de los datos fue realizado mediante test de Shapiro-Wilk. Las estadísticas descriptivas se expresaron como valor absoluto y porcentaje para las variables categóricas, mientras que las variables continuas fue- 
ron expresadas como media \pm desviación estándar o mediana (p25-p75) según distribución normal. Los registros con datos faltantes o incompletos fueron excluidos en el análisis de la característica donde no se poseía información (análisis de casos disponibles), tanto por la naturaleza descriptiva del estudio, tipo de información faltante, como por la falta de una relación causal entre el determinante de los datos perdidos (pérdida por omisión accidental en registro clínico) con las respectivas características de interés ${ }^{15,16}$. Debido al tipo y tamaño de la muestra, para comparar la frecuencia de desenlaces adversos según la gravedad de la cirrosis y tipo de cirugía (urgencia/electiva), se utilizó un criterio de importancia clínico (no estadístico) y se reportó gráficamente, sin "p-values"17,18. Los análisis fueron realizados en el programa Stata versión 12.0 (StataCorp 2011, Texas, Estados Unidos de Norteamérica).

\section{Aspectos éticos}

El estudio fue aprobado por el Comité de Ética Científico del Hospital Clínico Universidad de Chile el cual sigue la declaración de Helsinki.

\section{Resultados}

Durante el período de estudio se sometieron a cirugía 102 pacientes cirróticos en nuestro centro. Entre las características clínicas previos a la cirugía destacaban una media de edad 59,6 $\pm 11,3$ años, 32 de ellos mayores de 65 años (31\%), con predominio masculino (52\%). Las principales etiologías del daño hepático fueron esteatohepatitis no alcohólica (31\%), cirrosis alcohólica (28\%), hepatitis autoinmune (14\%) y etiología no identificada en $8 \%$ (Tabla 1).

Se observa una alta proporción de pacientes con daño hepático avanzado (Tabla 2): $50 \%$ con Child-Pugh B o C, MELD con mediana de 11, $25 \%$ de los pacientes con MELD igual o mayor a 15 , presencia de ascitis en $29 \%$ de los casos, plaquetopenia menor a $100.000 \mathrm{~mm}^{3}$ en $67,4 \%$, bilirrubinemia mayor a $1,5 \mathrm{mg} / \mathrm{dL}$ en $53 \%$, hipoalbuminemia menor a $3 \mathrm{mg} / \mathrm{dL}$ en $38,5 \%$ de ellos e INR mayor a 1,5 en $36,6 \%$. Las comorbilidades frecuentes fueron hipertensión arterial y diabetes mellitus (35,3\% y $21,3 \%$ respectivamente; Tabla 1). La Tabla suplementaria 1 muestra las variables con datos faltantes.
Tabla 1. Características basales de los pacientes operados

\begin{tabular}{lc|}
\hline Características & Media \pm DE; n (\%) \\
\hline Edad (años) & $59,59 \pm 11,28$ \\
Sexo masculino & $54(52,94 \%)$ \\
Etiología del daño hepático & \\
Alcohólica & $28(27,45 \%)$ \\
EHNA & $32(31,37 \%)$ \\
Virus hepatitis B & $4(3,92 \%)$ \\
Virus hepatitis C & $5(4,90 \%)$ \\
Hepatitis autoinmune & $14(13,73 \%)$ \\
Cirrosis biliar primaria & $6(5,88 \%)$ \\
Otras etiologías conocidas & $5(4,90 \%)$ \\
Desconocida & $8(7,84 \%)$ \\
Comorbilidades & $36(35,29 \%)$ \\
Hipertensión arterial & $32(31,37 \%)$ \\
Diabetes mellitus & $7(6,86 \%)$ \\
LCFA & $9(8,82 \%)$ \\
Hipotiroidismo & $7(6,86 \%)$ \\
Cáncer (cualquiera) & $31(30,39 \%)$ \\
Otras &
\end{tabular}

EHNA: Esteatohepatitis no alcohólica, LCFA: Limitación crónica del flujo aéreo. DE: Desviación estándar, p25-p75: rango intercuartílico.

Tabla 2. Gravedad de la cirrosis y condiciones asociadas

\begin{tabular}{|lc|}
\hline Características & $\begin{array}{c}\text { Mediana } \\
\text { (p25-p75); n (\%) }\end{array}$ \\
\hline Escala Child-Pugh * & B (A - B) \\
A & $44(50 \%)$ \\
B & $25(28,41 \%)$ \\
\hline Escala MELD* & $19(21,59 \%)$ \\
\hline Ascitis* & $11(10-15)$ \\
\hline Encefalopatía* & $27(29,35 \%)$ \\
\hline Anemia (Hb < 12 o $13 \mathrm{mg} / \mathrm{dL})^{*, a}$ & $14(14 \%)$ \\
\hline Plaquetopenia $(<100.000 \mathrm{~mm})^{*}$ & $39(41,49 \%)$ \\
\hline Bilirrubinemia elevada $(>1,5 \mathrm{mg} / \mathrm{dL})^{*}$ & $62(67,39 \%)$ \\
\hline Hipoalbuminemia $(<3,0 \mathrm{mg} / \mathrm{dL})^{*}$ & $35(33,41 \%)$ \\
\hline INR elevado $(>1,5)^{*}$ & $35(36,56 \%)$ \\
\hline Falla renal (creatinina $>1,5 \mathrm{mg} / \mathrm{dL})^{*}$ & $6(6,25 \%)$ \\
\hline
\end{tabular}

DE: Desviación estándar, p25-p75: rango intercuartílico. aValor de hemoglobina para mujeres y hombres, respectivamente, *Variable con datos faltantes. 
Tabla Suplementaria 1. Variables con datos faltantes en el estudio

\begin{tabular}{|c|c|}
\hline Variable & $\begin{array}{c}\text { Datos faltantes, } \\
\text { n (\%) }\end{array}$ \\
\hline Escala Child-Pugh & $14(13,73 \%)$ \\
\hline Escala MELD & $19(18,63 \%)$ \\
\hline Ascitis & $10(9,80 \%)$ \\
\hline Encefalopatía & $2(1,96 \%)$ \\
\hline Anemia $(\mathrm{Hb}<12$ o 13 mg/dL) & $8 \quad(7,84 \%)$ \\
\hline Plaquetopenia $\left(<100.000 \mathrm{~mm}^{3}\right)$ & $10 \quad(9,80 \%)$ \\
\hline Bilirrubinemia elevada ( $>1,5 \mathrm{mg} / \mathrm{dL}$ ) & $14(13,73 \%)$ \\
\hline Hipoalbuminemia (< 3,0 mg/dL) & $11(10,78 \%)$ \\
\hline INR elevado $(>1,5)$ & $6 \quad(5,88 \%)$ \\
\hline Falla renal (creatinina $>1,5 \mathrm{mg} / \mathrm{dL}$ ) & $6(5,88)$ \\
\hline Riesgo anestésico ASA & $5 \quad(4,90 \%)$ \\
\hline Requerimiento de diálisis & $2(1,96 \%)$ \\
\hline Transfusión en pabellón & $3(2,94 \%)$ \\
\hline
\end{tabular}

a: Valor de hemoglobina para mujeres y hombres, respectivamente.

Respecto a la cirugía practicada, correspondió a cirugía electiva en $71 \%$ de los casos, siendo más frecuente la cirugía abdominal (86,3\% del total), en particular la de hernia y patología biliar, en concordancia con los diagnósticos prequirúrgicos más frecuentes que motivaron estas cirugías (hernias y litiasis biliar, Tabla 3 ). De las 30 cirugías de urgencia, las principales fueron hernioplastía $(23,3 \%)$, resección intestinal $(23,3 \%)$ y laparotomía exploradora $(16,7 \%)$. El riesgo anestésico fue elevado, con una mediana de 3 en la escala de ASA, y $52 \%$ de los pacientes con ASA 3 o mayor, recibiendo casi la totalidad (98\%) anestesia general durante la cirugía (Tabla 3 ).

El desarrollo de complicaciones luego de la cirugía fue una condición frecuente, observada en $61,8 \%$ de los sujetos cirróticos. La Tabla 4 detalla la frecuencia de cada desenlace adverso asociado a las cirugías practicadas, agrupándolos en complicaciones mayores o con disfunción orgánica $(37,3 \%)$, asociados al hígado o cirrosis $(39,2 \%)$, o misceláneos $(33,3 \%)$. A nivel particular, las complicaciones más frecuentes fueron el ingreso a UPC $(25,5 \%)$, aumento de la ascitis $(22,6 \%)$, aumento de la encefalopatía
Tabla 3. Características de la cirugía practicada

\begin{tabular}{|c|c|}
\hline Característica & $\begin{array}{c}\text { Mediana } \\
\text { (p25-p75); n (\%) }\end{array}$ \\
\hline \multicolumn{2}{|l|}{ Diagnóstico prequirúrgico } \\
\hline Hernia umbilical & $10 \quad(9,80 \%)$ \\
\hline Hernia inguinal & $6(5,88 \%)$ \\
\hline Hernia incisional & $6(5,88 \%)$ \\
\hline Otro tipo de hernia & $5 \quad(4,90 \%)$ \\
\hline Colelitiasis & $24(23,53 \%)$ \\
\hline Colecistitis & $5 \quad(4,90 \%)$ \\
\hline Coledocolitiasis & $4 \quad(3,92 \%)$ \\
\hline Perforación de víscera hueca & $3(2,94 \%)$ \\
\hline Obstrucción intestinal & $6(5,88 \%)$ \\
\hline Hepatocarcinoma & $13(12,75 \%)$ \\
\hline Neoplasia abdomen o pelvis & $4 \quad(3,92 \%)$ \\
\hline Empiema pleural & $2(1,96 \%)$ \\
\hline Hematoma subdural & $2(1,96 \%)$ \\
\hline Otros & $12(11,76 \%)$ \\
\hline \multicolumn{2}{|l|}{ Cirugía realizada } \\
\hline Hernioplastía & $26(25,49 \%)$ \\
\hline Colecistectomía laparoscópica & $22(21,57 \%)$ \\
\hline Colecistectomía laparoscópica + ClO & $10(9,80 \%)$ \\
\hline Hepatectomía segmentaria & $13(12,75 \%)$ \\
\hline Laparatomía exploradora & $8(7,84 \%)$ \\
\hline Resección intestinal & $9(8,82 \%)$ \\
\hline Videotoracoscopía & $2(1,96 \%)$ \\
\hline Drenaje hematoma subdural & $2(1,96 \%)$ \\
\hline Otras & $10 \quad(9,80 \%)$ \\
\hline Cirugía de urgencia & $30(29,41 \%)$ \\
\hline Riesgo anestésico ASA* & $3(2-3)$ \\
\hline 1 & $4 \quad(4,12 \%)$ \\
\hline 2 & $44(45,36 \%)$ \\
\hline 3 & $41(42,27 \%)$ \\
\hline 4 & $7 \quad(7,22 \%)$ \\
\hline 5 & $1 \quad(1,03 \%)$ \\
\hline Anestesia general & $100(98,04 \%)$ \\
\hline
\end{tabular}

CIO: Colangiografía intraoperatoria. *Variable con datos faltantes.

hepática $(21,6 \%)$, y falla renal aguda $(23,5 \%)$. Por otra parte, la frecuencia de infecciones fue de $13,7 \%$, siendo más de la mitad de los casos de foco abdominal.

La necesidad de segunda cirugía fue un evento infrecuente (3,9\%), sin embargo, en dos de ellos se realizó trasplante hepático. La muerte intra- 
Tabla 4. Desenlaces adversos asociados a la cirugía practicada

\begin{tabular}{|lc|}
\hline Desenlaces adversos & n (\%) \\
\hline Morbimortalidad global & $63(61,76 \%)$ \\
\hline Complicación mayor/disfunción orgánica & $38(37,25 \%)$ \\
Muerte intrahospitalaria & $6(5,88 \%)$ \\
Ingreso a Unidad de Paciente Crítico & $26(25,49 \%)$ \\
Falla ventilatoria & $11(10,78 \%)$ \\
Falla renal aguda & $24(23,53 \%)$ \\
Requerimiento de diálisis* & $5(5 \%)$ \\
Segunda cirugía & $4(3,92 \%)$ \\
Trasplante hepático & $2(1,96 \%)$ \\
Asociado al hígado o cirrosis & $40(39,22 \%)$ \\
Sangrado variceal & $2(1,96 \%)$ \\
Aumento de ascitis & $23(22,55 \%)$ \\
Aumento de encefalopatía & $22(21,57 \%)$ \\
Hepatitis isquémica & $2(1,96 \%)$ \\
Misceláneos & $34(33,33 \%)$ \\
Transfusión en pabellón* & $11(11,11 \%)$ \\
Sangrado postoperatorio (no variceal) & $13(12,75 \%)$ \\
Infección en el postoperatorio & $14(13,73 \%)$ \\
Respiratoria & $3(21,43 \%)^{a}$ \\
Urinaria & $2(14,29 \%)^{a}$ \\
Abdominal & $8(57,14 \%)^{a}$ \\
Piel & $1(7,14 \%)^{a}$ \\
\hline
\end{tabular}

${ }^{a}$ Del total de pacientes con infecciones. *Variable con datos faltantes. hospitalaria ocurrió en seis pacientes (5,9\%), tres de ellos en relación a sangrado y tres asociados a infecciones. En la Tabla 5 se detallan las características y causa de muerte de los pacientes fallecidos.

Al comparar exploratoriamente la frecuencia de desenlaces adversos según la gravedad de la cirrosis o tipo de cirugía (Figura 1), los pacientes con Child-Pugh C, comparados con aquellos Child-Pugh A y B, presentaron mayor morbimortalidad global, mayor frecuencia de complicaciones graves y mayor frecuencia de complicaciones misceláneas (Figura 1A). A su vez, se observó un aumento en la morbimortalidad global, complicaciones mayores y misceláneas en relación al aumento del MELD, mostrando peores desenlaces aquellos pacientes con MELD > 15 (Figura 1B). Finalmente, en la Figura 1C se observa que los cirróticos sometidos a cirugía de urgencia desarrollaron más complicaciones quirúrgicas que los sometidos a cirugías electivas, en términos de morbimortalidad global, complicaciones mayores y desenlaces misceláneos. No hubo diferencias clínicas significativas en el desarrollo de complicaciones hepáticas al comparar por Child-Pugh, MELD o tipo de cirugía.

Tabla 5. Características y causa de muerte de los pacientes fallecidos

\begin{tabular}{|c|c|c|c|c|c|c|c|c|c|c|}
\hline Edad & $\begin{array}{c}\text { Diagnós- } \\
\text { tico }\end{array}$ & Cirugía & $\begin{array}{c}\text { Urgencia } \\
\text { quirúrgica }\end{array}$ & $\begin{array}{l}\text { Child- } \\
\text { Pugh }\end{array}$ & MELD & $\begin{array}{c}\text { Plaquetas } \\
\left(x \mathrm{~mm}^{3}\right)\end{array}$ & Ascitis & Enc. & ASA & $\begin{array}{l}\text { Causa de } \\
\text { muerte }\end{array}$ \\
\hline 58 & $\begin{array}{c}\text { Perforación } \\
\text { víscera } \\
\text { hueca }\end{array}$ & $\begin{array}{l}\text { Laparotomía } \\
\text { exploradora }\end{array}$ & Sí & C & 20 & 92.000 & G 1 & G 3 & 4 & $\begin{array}{l}\text { Coagulopatía } \\
\text { refractaria } \\
\text { con sangrado } \\
\text { activo }\end{array}$ \\
\hline 52 & $\begin{array}{l}\text { Tumor } \\
\text { gástrico }\end{array}$ & $\begin{array}{c}\text { Gastrectomia } \\
\text { total }\end{array}$ & No & B & 15 & 45.000 & G 0 & No & 3 & $\begin{array}{l}\text { Sepsis - } \\
\text { Disfunción } \\
\text { multiorgánica }\end{array}$ \\
\hline 54 & $\begin{array}{l}\text { Hernia } \\
\text { incisional }\end{array}$ & $\begin{array}{c}\text { Hernioplastía } \\
\text { con malla }\end{array}$ & Sí & C & 23 & 77.000 & G 2 & G 1 & 4 & $\begin{array}{l}\text { Sepsis - } \\
\text { Disfunción } \\
\text { multiorgánica }\end{array}$ \\
\hline 61 & $\begin{array}{l}\text { Colecistitis } \\
\text { aguda }\end{array}$ & $\begin{array}{l}\text { Colecistectomía } \\
\text { laparoscópica }\end{array}$ & Sí & B & 16 & 93.000 & G 0 & No & 3 & $\begin{array}{l}\text { Shock } \\
\text { hemorrágico }\end{array}$ \\
\hline 65 & $\begin{array}{l}\text { Hernia } \\
\text { umbilical } \\
\text { atascada }\end{array}$ & $\begin{array}{l}\text { Laparotomia } \\
\text { exploradora más } \\
\text { hernioplastía }\end{array}$ & Sí & B & 16 & 120.000 & G 1 & No & 3 & Shock séptico \\
\hline 55 & $\begin{array}{c}\text { Hematoma } \\
\text { subdural }\end{array}$ & $\begin{array}{l}\text { Vaciamiento } \\
\text { hematoma } \\
\text { subdural }\end{array}$ & Sí & C & 35 & 70.000 & G 2 & G 1 & 4 & $\begin{array}{l}\text { Coagulopatía } \\
\text { refractaria } \\
\text { con sangrado } \\
\text { activo }\end{array}$ \\
\hline
\end{tabular}

Enc: Encefalopatia hepática, ASA: Riesgo anestésico según ASA, G: Grado. 


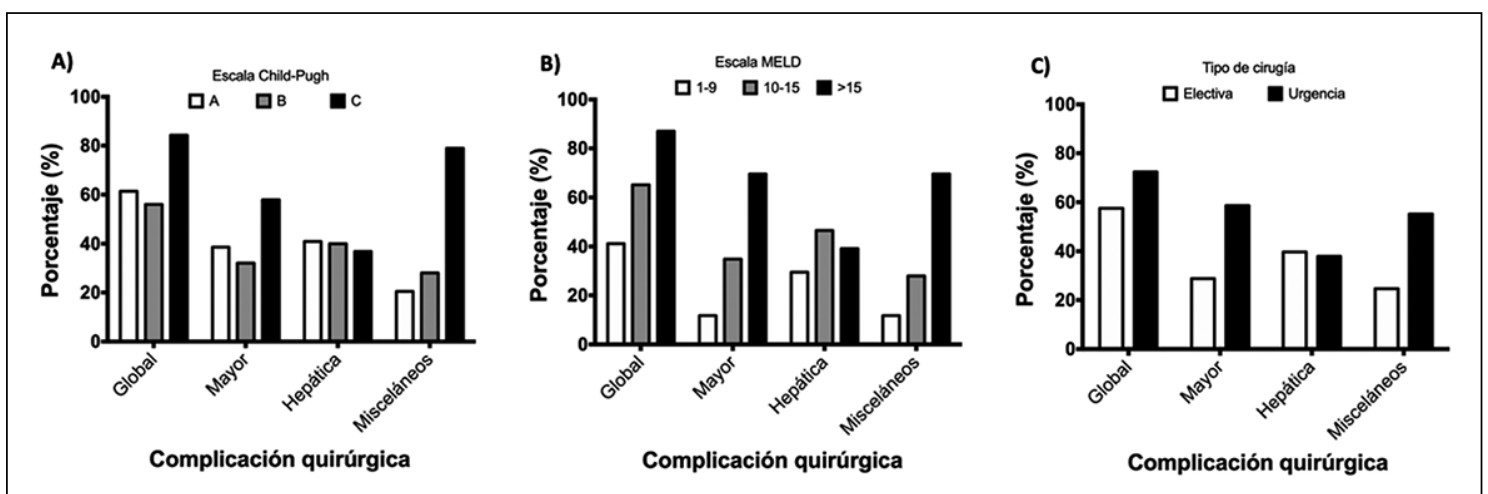

Figura 1. Complicaciones quirúrgica según gravedad de la cirrosis y tipo de cirugía. Se compara el porcentaje de morbimortalidad global, complicaciones mayores/disfunción orgánica, complicaciones hepáticas o relacionadas a la cirrosis y desenlaces misceláneos. En A) se compara según la escala de Child-Pugh, en B) según el puntaje de MELD, y en C) cirugía de urgencia versus cirugía abdominal.

\section{Discusión}

Este trabajo reporta las características y desenlaces clínicos asociados a la cirugía en cirróticos de un centro de salud terciario en Chile, lo que constituye una información relevante y escasamente informada a nivel nacional y en Latinoamérica.

En nuestra experiencia como centro, destaca la alta carga de morbilidad de los pacientes, previo a la cirugía y la gravedad de la enfermedad hepática, gran parte de ellos en etapa avanzada. Por otro lado, las cirugías practicadas fueron en su mayor parte electivas, relacionadas al abdomen o digestiva, y con un riesgo anestésico considerable. En concordancia, en esta cohorte de pacientes cirróticos se reporta una alta morbimortalidad operatoria global $(61,8 \%)$, con una elevada frecuencia de complicaciones graves (representadas en $37,3 \%$ de complicaciones mayores y $25 \%$ de ingresos a UPC), pero con una baja mortalidad intrahospitalaria $(5,9 \%)$.

\section{Características basales, gravedad del daño hepático y complicaciones quirúrgicas}

Dentro de las características basales destaca una alta prevalencia de hipertensión arterial, diabetes mellitus y otras comorbilidades no relacionadas a la cirrosis (Tabla 1). En la literatura se menciona que hasta $40 \%$ de los cirróticos tienen alguna comorbilidad, que aumentarían la mortalidad ${ }^{13}$. Un estudio danés sobre comorbilidades en cerca de 13.000 pacientes con daño hepático crónico $^{19}$ señala como principal comorbilidad a la diabetes mellitus, con una baja prevalencia de hipertensión arterial, la que se consideró en caso de complicaciones asociadas. Por otra parte, la prevalencia de limitación crónica del flujo aéreo (LCFA) y cáncer son similares a nuestro registro ${ }^{13,19}$.

En términos de la gravedad del daño hepático crónico, los pacientes incluidos en nuestro estudio presentaron puntajes de Child-Pugh A (50\%), seguido de $\mathrm{B}(28,4 \%)$ y C $(21,6 \%)$ y una mediana de MELD de 11, con $25 \%$ de pacientes con puntaje $>15$, lo que representa una gravedad mayor a lo reportado en estudios previos ${ }^{8,20,21}$. Lo anterior es relevante, ya que una de las herramientas utilizada para valorar el riesgo quirúrgico en el cirrótico han sido los puntajes de Child-Pugh y MELD ${ }^{1-4}$. Incluso en pacientes con Child-Pugh A, la morbilidad perioperatoria está aumentada y tendría relación con el grado de hipertensión portal ${ }^{3}$. En concordancia, la elevada morbimortalidad observada en nuestro estudio (Tabla 4) podría deberse en parte a la gravedad del daño hepático de los pacientes estudiados y su comorbilidad asociada.

Corroborando lo reportado en otras cohortes $^{6-8,10}$, en el análisis exploratorio se observaron peores desenlaces quirúrgicos en pacientes con enfermedad hepática más avanzada, determinados por las escalas de Child-Pugh y MELD (Figura 1A y 1B), no siendo ninguno de los pacientes fallecidos Child A (Tabla 5). A pesar de esta asociación, cabe destacar que la mayoría de las complicaciones perioperatorias observadas en nuestro estudio están relacionadas con el sangrado y con complicaciones hepáticas o asociadas a la cirrosis (Tabla 4), 
complicaciones que no mostraron asociación con la gravedad de la enfermedad ni con el tipo de cirugía (Figura 1).

\section{Tipos de cirugías, riesgo anestésico y desenlaces adversos}

Del total de cirugías realizadas, se observó $29,4 \%$ de cirugías de urgencia, casi el triple de lo reportado por otros estudios ${ }^{6,8,9}$. Es sabido que la cirugía de urgencia se asocia a mayor mortalidad, tanto en el paciente cirrótico como en el no cirrótico $^{1-4}$. En particular, en nuestra muestra, cinco de los seis pacientes fallecidos correspondían a pacientes intervenidos de urgencia, lo que representa una mortalidad de $16,6 \%$ en este grupo, casi tres veces la mortalidad de la cohorte total, y más de 10 veces la mortalidad observada en el grupo de cirugía electiva (1/72). Además, se evidenció una mayor morbimortalidad global, complicaciones mayores y misceláneas en los pacientes con cirugía de urgencia versus electiva (Figura 1C). Esto se observa también en otras series, como Neeff y cols. ${ }^{20}$, quienes describieron una mortalidad total de $27,5 \%$ en cirugías no hepáticas, donde la mayor mortalidad se concentraba en el grupo de pacientes de urgencia ( $8,7 \%$ versus $47 \%)$ y Kim y cols. ${ }^{21}$, quienes reportaron una mortalidad de $35,8 \%$ en paciente que fueron sometidos a cirugía de urgencia.

El riesgo anestésico objetivado con la escala ASA es un buen predictor de complicaciones postoperatorias. En nuestra muestra, la mitad de los pacientes presentaron ASA 3 o mayor, lo que es bastante similar a lo reportado en estudios previos en cirróticos sometidos a cirugía abdominal ${ }^{7,10}$.

En relación a los diagnósticos preoperatorios, los pacientes con daño hepático crónico se caracterizan generalmente por presentar mayor riesgo de hernias de la pared abdominal y colelitiasis ${ }^{21}$. Esta última condición, además, presenta una alta prevalencia en la población chilena ${ }^{22,23}$. Es así como podemos ver en esta cohorte que más de $50 \%$ de las indicaciones quirúrgicas correspondía a patología herniaria o de la vía biliar. En este contexto es importante evaluar la vía de abordaje quirúrgico en cirugías abdominales, ya que se ha reportado una menor morbimortalidad con el abordaje laparoscópico ${ }^{24}$. En nuestro centro, la laparoscopía representa el estándar de cuidado, falleciendo solo un paciente que fue sometido a cirugía laparoscópica (Tabla 5).
Finalmente, si bien la frecuencia de complicaciones no fatales fue similar a lo reportado por otros estudios, la mortalidad observada en nuestro estudio $(5,9 \%)$ fue menor que lo informado en estudios de cirugía general ${ }^{9}$. Esto podría explicarse en parte porque los principales motivos quirúrgicos fueron la patología herniaria y biliar, las que han mostrado tener mortalidades más bajas que otras cirugías, y cercanas a las observadas en nuestra cohorte ${ }^{9,25,26}$.

\section{Limitaciones del estudio}

La principal limitación de nuestro estudio deriva de su diseño, basado en un registro histórico, que no incluye todos los tipos de cirugía, con presencia de algunos datos faltantes por paciente, un muestro no aleatorio y con datos de un único centro. Además, al no ser un estudio longitudinal, no es posible estimar la incidencia de ocurrencia de los eventos ni su comportamiento en el tiempo. A su vez, nuestra experiencia incluye un alto porcentaje de cirugía abdominal (86\%), por lo que otros tipos de cirugía y sus desenlaces se encuentran pobremente representadas.

Todo lo anterior, impide la extrapolación de los datos y el análisis inferencial, por lo que la información presentada debe tomarse como una referencia descriptiva, importante para la realidad local, pero que debe ser confirmada en futuros estudios longitudinales, multicéntricos y con mayor tamaño muestral.

\section{Conclusión}

Los cirróticos sometidos a cirugía presentan una alta carga de morbilidad y elevada tasa de complicaciones postquirúrgicas, a pesar de la baja mortalidad de nuestra serie. Es necesario ampliar la evidencia disponible en Latinoamérica sobre la cirugía en el paciente cirrótico, en la que una adecuada estratificación del riesgo operatorio es de suma importancia para conseguir mejores resultados.

\section{Referencias}

1. Nicoll A. Surgical risk in patients with cirrhosis. J Gastroenterol Hepatol 2012; 27 (10): 1569-75.

2. Friedman LS. Surgery in the patient with liver disease. 
Trans Am Clin Climatol Assoc 2010; 121: 192-204; discussion 205.

3. Hackl C, Schlitt HJ, Renner P, Lang SA. Liver surgery in cirrhosis and portal hypertension. World J Gastroenterol 2016; 22 (9): 2725-35.

4. Prenner S, Ganger D. Risk Stratification and Preoperative Evaluation of the Patient With Known or Suspected Liver Disease. Clin Liver Dis (Hoboken) 2016; 7(5): 101-5.

5. Gelman S. General anesthesia and hepatic circulation. Can J Physiol Pharmacol 1987; 65 (8): 1762-79.

6. Teh SH, Nagorney DM, Stevens SR, Offord KP, Therneau TM, Plevak DJ, et al. Risk Factors for Mortality After Surgery in Patients With Cirrhosis. Gastroenterology 2007; 132 (4): 1261-9.

7. Telem DA, Schiano T, Goldstone R, Han DK, Buch KE, Chin EH, et al. Factors That Predict Outcome of Abdominal Operations in Patients With Advanced Cirrhosis. Clin Gastroenterol Hepatol 2010; 8 (5): 451-7.

8. Kim SY, Yim HJ, Park SM, Kim JH, Jung SW, Kim JH, et al. Validation of a Mayo post-operative mortality risk prediction model in Korean cirrhotic patients. Liver Int 2011; 31 (2): 222-8.

9. de Goede B, Klitsie PJ, Lange JF, Metselaar HJ, Kazemier G. Morbidity and mortality related to non-hepatic surgery in patients with liver cirrhosis; A systematic review. Best Pract Res Clin Gastroenterol 2012; 26 (1): 47-59.

10. Costa BP, Castro Sousa F, Serôdio M, Carvalho C. Value of MELD and MELD-Based Indices in Surgical Risk Evaluation of Cirrhotic Patients: Retrospective Analysis of 190 Cases. World J Surg 2009; 33 (8): 1711-9.

11. Tsochatzis EA, Bosch J, Burroughs AK. Liver cirrhosis. Lancet 2014; 383 (9930): 1749-61.

12. Salvalaggio PR, Caicedo JC, de Albuquerque LC, Contreras A, Garcia VD, Felga GE, et al. Liver Transplantation in Latin America. Transplantation 2014; 98 (3): 241-6.

13. Jepsen P. Comorbidity in cirrhosis. World J Gastroenterol 2014; 20 (23): 7223-30.

14. Mokdad AA, López AD, Shahraz S, Lozano R, Mokdad $\mathrm{AH}$, Stanaway J, et al. Liver cirrhosis mortality in 187 countries between 1980 and 2010: a systematic analysis.
BMC Med 2014; 12: 145.

15. Ibrahim JG, Chu H, Chen M-H. Missing data in clinical studies: issues and methods. J Clin Oncol 2012; 30 (26): 3297-303.

16. Daniel RM, Kenward MG, Cousens SN, De Stavola BL. Using causal diagrams to guide analysis in missing data problems. Stat Methods Med Res 2012; 21 (3): 243-56.

17. Bangdiwala SI. To P or not to P. Int J Inj Contr Saf Promot 2013; 20 (4): 394-6.

18. Wasserstein RL, Lazar NA. The ASA's Statement on $p$ -Values: Context, Process, and Purpose. Am Stat 2016; 70 (2): 129-33.

19. Jepsen P, Vilstrup H, Lash TL. Development and Validation of a Comorbidity Scoring System for Patients With Cirrhosis. Gastroenterology 2014; 146 (1): 147-56.

20. Neeff HP, Streule GC, Drognitz O, Tittelbach-Helmrich D, Spangenberg H-C, Hopt UT, et al. Early mortality and long-term survival after abdominal surgery in patients with liver cirrhosis. Surgery 2014; 155 (4): 623-32.

21. Kim SH, Han YD, Lee JG, Kim DY, Choi SB, Choi GH, et al. MELD-Based Indices as Predictors of Mortality in Chronic Liver Disease Patients Who Undergo Emergency Surgery with General Anesthesia. J Gastrointest Surg 2011; 15 (11): 2029-35.

22. Lammert F, Gurusamy K, Ko CW, Miquel J-F, Méndez-Sánchez N, Portincasa P, et al. Gallstones. Nat Rev Dis Prim 2016; 2: 16024.

23. Latorre SG, Ivanovic-Zuvic SD, Corsi SÓ, Valdivia CG, Margozzini MP, Olea OR, et al. Cobertura de la estrategia preventiva de cáncer de vesícula biliar en Chile: Resultados de la Encuesta Nacional de Salud 2009-2010. Rev Med Chile 2015; 143 (2): 158-67.

24. Rai R, Nagral S, Nagral A. Surgery in a Patient with Liver Disease. J Clin Exp Hepatol 2012; 2 (3): 238-46.

25. Pandey CK, Karna ST, Pandey VK, Tandon M, Singhal A, Mangla V. Perioperative risk factors in patients with liver disease undergoing non-hepatic surgery. World J Gastrointest Surg 2012; 4 (12): 267-74.

26. López-Delgado JC, Ballus J, Esteve F, Betancur-Zambrano NL, Corral-Velez V, Mañez R, et al. Outcomes of abdominal surgery in patients with liver cirrhosis. World J Gastroenterol 2016; 22 (9): 2657-67. 